\title{
Aligning the chemical engineering curriculum to a common problem-solving strategy
}

\section{Prof. Nicolas Hudon, Queen's University}

Dr Nicolas Hudon is an Assistant Professor in the Department of Chemical Engineering at Queen's University (Canada) since 2016. His teaching activities are mainly concerned with second-year fundamental courses. He is the recipient of the 2019 Carolyn Small Award for teaching innovation from the Faculty of Applied Sciences and Engineering at Queen's University.

\section{Dr. Louise Meunier P.Eng., Queen's University}

Dr. Meunier studied mechanical engineering and worked for twenty years as an aerospace engineering officer in the Royal Canadian Air Force. She then completed a PhD in chemical engineering specializing in environmental chemistry. Dr. Meunier's research focuses on the toxicity of contaminants in soils and mine tailings, and on environmental and human health risks associated with exposure to contaminants in water and soil. These investigations include research on inorganic and organic pollutants, as well as novel materials (e.g. nanoparticles, graphene), and contaminant mixtures. For contaminated soils, tailings, and leachates, research results are used to reduce the costs associated with remediation by focusing cleaning efforts to areas where risk has been identified. In the study of novel materials, this research informs the design of new processes and products, with the goals of protecting human and environmental health. Dr. Meunier works in collaboration industrial and academic experts involved in various aspects of interdisciplinary environmental engineering research. Dr. Meunier is also involved in engineering education research; her interests include improving problem-solving and resilience abilities in engineering students, and incorporating innovative teaching approaches in the engineering curriculum. 


\title{
Aligning the chemical engineering curriculum to a common problem-solving strategy
}

\author{
Nicolas Hudon and Louise Meunier \\ \{nicolas.hudon,louise.meunier\}@queensu.ca \\ Department of Chemical Engineering \\ Queen's University \\ Kingston, ON, Canada
}

\begin{abstract}
This aim of this project is to improve students' abilities in solving chemical engineering problems by implementing, in the chemical engineering curriculum, a consistent and effective approach to problem solving. The key concept is to foster in students an understanding of the structure of chemical engineering problems from early courses, and to implement the same approach throughout their undergraduate training by reinforcing a consistent methodology. The project is supported by surveys on students' confidence level in problem solving which are used to adapt our teaching to students' needs. In the present contribution, an overview of the project is given and potential applications of a proposed concept map throughout the curriculum is discussed.
\end{abstract}

\section{Introduction}

The ability to solve problems is key to the success of engineers and engineering students alike $^{1}$. However, it has been long recognized that teaching and reinforcing problem solving are complex tasks. Many students fail to apply effective problem-solving techniques in tutorials, assignments, and exams, especially in fundamental science courses. Moreover, except in design-oriented activities, common problem-solving techniques are usually not re-enforced nor consistently presented between undergraduate courses. Most problem-solving techniques surveyed in the literature are not targeted to chemical engineering and adapting a generic technique to chemical engineering problems is not straightforward. Instructors and students alike often interpret poor results in problem solving as a lack of understanding of the fundamentals or as gaps in mathematical knowledge. Publications on problem solving are extensive in engineering education literature and education literature ${ }^{2,3}$; however, no clear approach is widely recognized as a foolproof method to design teaching and training activities that develop and reinforce problem-solving skills. The motivation behind this educational project is to propose a consistent approach for students to organize data and theory and to effectively improve their problem-solving skills early in the undergraduate curriculum. This project was initiated by two 
instructors involved in a sequence of fundamental chemical engineering second-year courses: Course $\mathrm{A}$ is the fundamental mass and energy balances introductory course delivered to students during the Fall term of their second year of engineering studies; and, Course B is the first course of chemical engineering thermodynamics, taught during the Winter term of second year. The key outcome in proposing an effective and consistent approach to problem solving early in the chemical engineering curriculum is to enhance students' learning experience by demystifying what they perceived as their main weaknesses when solving problems.

Developing problem-solving skills for (chemical) engineering students is covered extensively in engineering education literature, for example the McMaster Problem Solving Program $^{4}$ and the discussion presented in Chapter 5 of the book by Wankat and Oreovizc ${ }^{5}$. Following the analysis of survey results ${ }^{6}$ and based on a classical reference on problem-solving in mathematics ${ }^{7}$, one instructor involved in the project redesigned the second-year introduction to thermodynamics course by developing an extensive set of solved problems, including tutorials and video examples that the students can study at their own pace, focusing on the early stages of problem solving: understanding the problem, making a diagram, listing known and unknown variables, making an inventory of learned concepts, and devising a plan for solution. However, results of a subsequent survey revealed that, although students had improved their confidence in solving problems, the main perceived difficulty remains setting up and solving appropriate mathematical equations. This result is counter-intuitive, because early calculus and manipulation of simple algebraic equations are the main mathematical tools used in solving applied thermodynamic problems at this level. These skills are considered acquired knowledge that students are expected to have previously applied in first-year physics and calculus classes. This prompted both instructors involved in the project to re-consider the usual problem-solving techniques and to develop a more context-specific approach to chemical engineering problem solving. This resulted in the construction of a concept map ${ }^{8}$ presented in Figure 1. This concept map is introduced early to the second-year students to motivate all pedagogical activities. The concept map is centered on conservation laws (or balance laws) as a common organizing principle to chemical engineering problems. The proposed concept map can be related to previous efforts in the literature to enhance problem-solving skills for specific topics, notably in thermodynamics ${ }^{9,10}$, statics ${ }^{11,12}$, and electrical engineering circuits ${ }^{13}$. However, the present project goes beyond specific courses and seeks to enhance students' problem-solving skills by deconstructing chemical engineering problems; this approach is argued in the chemistry education literature ${ }^{14}$. It is extrapolated here with a clear objective of improving long-term abilities in solving problems ${ }^{15}$ by outlining the main features of chemical engineering problems. As reported in a previous contribution $^{8}$, instructors gradually shifted the focus of their problem-solving demonstrations to an approach through which general balances are systematically presented, and where all information is to be viewed through the lens of balance equations, as depicted in the proposed concept map (Figure 1). Currently ${ }^{16}$, the authors also seek to anchor the project through behaviorism and objectivism to increase students' awareness of the learning process ${ }^{17}$. In this contribution, the authors illustrate how the proposed concept map can be applied as a tool for curriculum alignment, i.e., the authors discuss how the same concept map can be used to devise solutions for problems of growing complexity in the chemical engineering curriculum. 
The manuscript is structured as follows. The concept map is presented first, and its usage in second-year courses is described, with a full thermodynamic example presented in Appendix A. The idea of implementing the concept map as a curriculum alignment tool is then proposed and discussed. Survey results outlining positive and negative effects of the concept map as a tool to design instruction activities for chemical engineering students are presented and discussed. Conclusions and areas for further developments are also given.

\section{Concept Map}

The concept map was proposed and discussed originally in a previous contribution by the authors ${ }^{8}$. It was conceived following the observation of a fallacy in engineering education: There is a general perception, perpetuated by students and instructors alike, that students are ill-equipped technically, i.e., that they do not possess the required mathematical knowledge to tackle upper-year engineering problems. Student surveys, as presented later in this contribution, and recent efforts by the Engineering Faculty at Queen's University, where this project is taking place to re-align mathematics instruction to correct identified gaps in mathematical proficiency in upper-year cohorts are evidences that this perception exists. However, both instructors involved in this educational project observed that such perception is unfounded, because the level of mathematical sophistication required in their courses are limited to linear algebra and early integral calculus.

Compounding this issue, the literature on engineering problem-solving methodologies ${ }^{6,5}$ is generally oriented toward open-problems (notably engineering design problems) or is too general and therefore unsuitable for junior-level engineering fundamental courses. A systematic approach to be presented to students and reinforced as early as possible in the instruction of fundamental discipline-oriented courses was therefore needed. The sought approach needed to be general enough to be applicable to a maximum of courses but specific enough for the students to find it useful in solving problems in tutorials, assignments, and exams.

Finally, in seeking effective problem-solving methodologies, the authors concluded that many techniques reported in the literature focus on early problem-solving steps (i.e., reading the problem, drawing a schematic of the problem, listing the necessary theory, etc.), but this accent is misplaced and the true obstacles are not addressed. Survey results ${ }^{8}$ indicate that the emphasis on early steps does not match what students perceive as the most difficult steps which are, in particular, setting up and solving the mathematical equations. Hence, a re-thinking of chemical engineering problems was initiated through the concept map (Figure 1).

At the center of the proposed concept map are the balance equations - for example mass, energy, and entropy balances in thermodynamics ${ }^{18}$. At the core of a chemical processes and systems course or a reactor analysis and design course, mass and energy balances apply, whereas for fluid mechanics problems, one would consider mass and momentum balances. Generally, the concept map is based on the fundamental realization that all chemical engineering courses require the application (and solution) of conservation laws. Hence, a consistent problem-solving methodology for chemical engineering problems must be centered on the conservation of extensive quantities. If the system is homogeneous, then the adequate framework requires the solution of Ordinary Differential Equations (ODEs), whereas Partial Differential Equations (PDEs) are to be considered for non-homogeneous systems. In both courses taught by the 


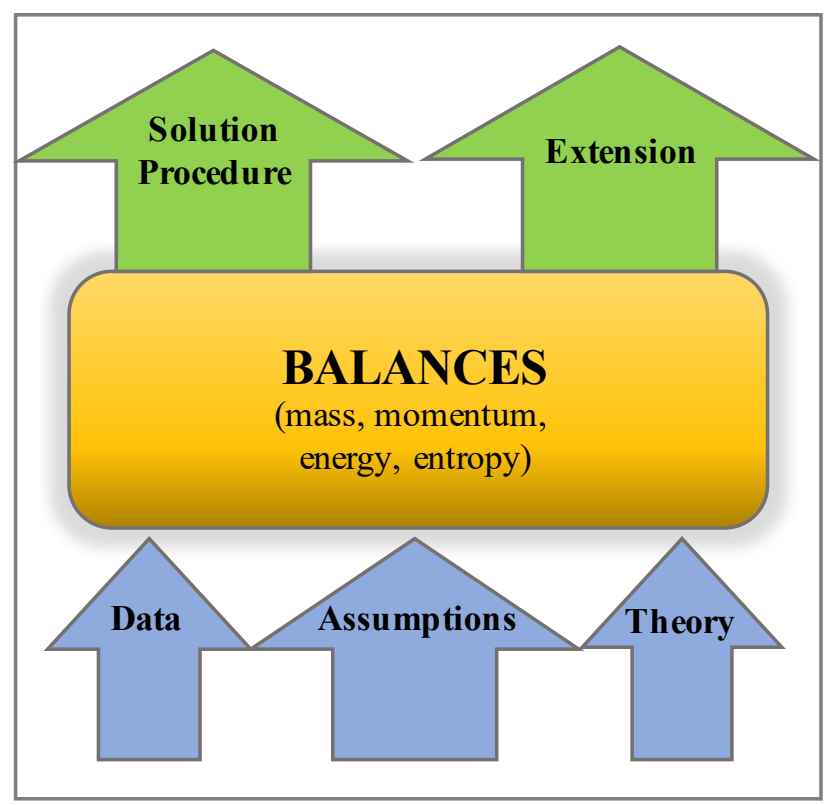

Figure 1: Concept map ${ }^{8}$

instructors involved with this project (introduction to energy and mass balances and introduction to thermodynamics), the proper mathematical framework consists in balance equations described by ODEs together with appropriate constitutive relationships.

Once the mathematical framework is established, balance equations are stated, in the general form, for each extensive quantity considered, as

$$
\text { Accumulation rate }=\text { rate in }- \text { rate out } \pm \text { rate consumed/produced, }
$$

and students can, by reading the problem statement, begin working on a solution using Assumptions, Theory, and provided Data/Relations. The mathematical form for each term in the above expression obviously depends on the course topic: balance equations for an homogeneous system in thermodynamics differ from balance equations in a non-homogeneous system in transport phenomena. These different mathematical frameworks impact the solution step by requiring different mathematical tools. However, overall, the structure of the problems remains essentially the same: conservation laws are central to chemical engineering problems. And the solution steps in the analysis of a given problem are essentially the same. For example, based on the theory and on the class of mathematical systems under study, students can cancel terms in the balance equations, e.g., setting the accumulation rate to zero if the problem is at steady state. Exploiting stated assumptions, constitutive relationships and specified constraints, students can also perform degrees-of-freedom analysis and devise a plan for solving the balance equations.

The authors of this contribution posit that through the process of writing general equations and working the solution first by simplifying balance equations, students become gradually more confident in solving these equations and extending their understanding by relaxing and/or questioning some assumptions. To them, the impact of an assumption is clear, because it 
translates directly into the resulting balance equations and in the solution procedure. This eases their progression from solving simple problems to more complex problems as a given course progresses, and this approach generally increases their confidence in solving problems. Discussions about degrees-of-freedom analysis and sequence of steps to solve specific problems can be naturally included in demonstrations as the resulting equations are developed explicitly.

In both second-year courses where the concept map has been in used for two years, this shift in instruction was adopted in many problem-solving activities: in-class examples and tutorial sessions, video examples, homework and supplementary problems. Systematically applying the concept map enables the instructors to teach chemical engineering material while avoiding formulae sheets, which tend to limit students solution thought process to patterns and templates. Indeed, when students rely on formulae sheets, it has been observed that some students guess the right formula to be used rather than think from first principle. To develop sound problem-solving abilities, students should be encouraged to focus on first principles rather than rely on formulae sheets. This approach is required to improve students' understanding of examples and tutorial activities, and, to gradually improve students' confidence in solving chemical engineering problems.

It should be noted that the concept map approach is a work in progress. The problem-solving methodology cannot be followed blindly; students must also develop skills in troubleshooting and recognizing wrong turns to correct mistakes along the way. Moreover, as highlighted in student surveys, the enhanced confidence in problem-solving skills does not extend beyond one course, hence the motivation to expand the proposed project beyond the second year. In other words, continuity in applying the concept map is key to develop, in chemical engineering students, long-lasting and effective problem-solving habits.

\section{Using the Concept Map in Curriculum Alignment}

The problem-solving methodology is systematically applied in all aspects of Courses A and $\mathrm{B}$, in which the authors of this contribution are involved (in-class examples, tutorials, assignments, online interactive exercises, and video examples). During the second year of implementation, special emphasis was placed on student awareness of the process of accessing acquired knowledge to solve each problem. Survey results from the first year of the project clearly demonstrated that "developing mathematical equations" is perceived by students as the most difficult step in problem solving. In the present section, we focus the discussion to curriculum alignment. We argue that, as problems evolve along the chemical engineering curriculum, it is possible for instructors to shift emphasis on specific elements of the concept map to enhance student comprehension of chemical engineering.

A detailed description of the pedagogical approach and activities for Courses A and B is presented in previous publication ${ }^{8}$. Briefly, Course A material is presented in a series of modules, starting from simple mass balances at steady state, then accounting for variations in energy and system fluctuations over time. In Course B, the first and second laws of thermodynamics are connected to the concept of balances, notably by adding the entropy balance. Course B is then 
based on the solution of a system of balance equations to which fundamental notions on constitutive relationships and degrees of freedom analysis are gradually incorporated. Throughout both Courses A and B, students are asked to derive solutions for systems of increasing complexity, both from the mathematical and the fundamental knowledge perspectives.

Survey results over two years of implementations of the concept map show some potential for the long-term impact of the proposed teaching strategies. However, it is clear that a crucial element is continuity of pedagogical approach and learning learning process. In particular, the barrier to be overcome in the next stages of this project is the "one course, one problem-solving strategy" attitude among many students. To this end, the authors propose some thoughts and potential opportunities to expand the concept map as a teaching design tool throughout the curriculum. The objective is to demonstrate that adapting instructors' perspectives on problems solving to advocate a general and consistent approach benefits students, who can then develop an understanding of the field and extrapolate their skills rather than apply them in a course-per-course approach.

The Concept Map for Second-year Courses

In second-year instruction, problems are generally closed, i.e., students are given all the data necessary to solve the problem under suitable assumptions. So far in this project, the approach was applied in the fundamental mass and energy balance course as well as the introductory thermodynamic course. The objective of examples and tutorial problems is generally to illustrate the theory. Homework and exam problems are generally constructed such that students demonstrate their understanding of the fundamentals of chemical engineering science. Thus, once the concept map is established and it is clear to the students that the mathematical framework for any problem is the solution of balance equations (Theory), then the emphasis in instruction should be placed on the following elements:

- Assumptions - Simplify the set of balance equations; and,

- Data - Solve the problem numerically.

Finding the data, for example using tables, is generally perceived as easy by the students once the context of solving balance equations is clear. Solutions are usually simple calculus and algebraic manipulations. In this context, problem extensions are often used by the instructor to generate motivations for and setup an introduction to subsequent elements of the theory to be covered in the class.

\section{The Concept Map for Third-year Courses}

In third year courses, students are exposed to more complex problems. For the expansion of the current project, reactor design and heat and mass transfer are the courses the authors have in mind. In general, because these courses involve ODEs and PDEs, the emphasis in problem-solving is gradually shifted to computational aspects of solution derivation. At the same time, problems evolve from closed to open. As such, students must state assumptions, investigate and find sources for data. Extensions become more important, and students are asked to reflect on their assumptions and solution method. Bringing students into the thought process required to 
take on these new responsibilities may be easier if the overall structure for problem-solving is consistent in a curriculum. In third year, emphasis in instruction should be placed on the following elements:

- Data and Assumptions - Decide on relevant assumptions and data sources;

- Solution procedure - Identify the best solution method (numerical or analytical); and,

- Extension - Understand potential problem variations (impact of assumptions on solution procesure)..

The Concept Map for Fourth-year Courses

A large part of the last year in chemical engineering is dedicated to design problems, which are open problems. Students often use specialized software products to solve the balance equations inherent to chemical engineering design projects. Again, it is argued that the concept map can be central to the problem-solving methodology, even in open-ended problems. As data, assumptions, and relevant theory are ill-defined, students must understand that, at the core of a chemical engineering problem reside balance equations. The concept map can also be used as a solution verification procedure (or as a back-of-the-envelope thinking procedure in the early stages of design). In fourth year, emphasis in instruction should be placed on the following elements:

- Theory - Identify the required balances to be developed;

- Data and Assumptions - Test different assumptions and data sources;

- Solution procedure - Identify the solution path and understand the impact of approximations; and,

- Extension - Understand the impact of assumptions on solution robustness.

\section{Surveys and Results}

In the past two years, students were invited to fill out a survey to assess their problem solving confidence level, perceptions, and abilities at intervals during their studies. At this point, data has been collected for two successive cohorts. Cohort 1 was assessed at the beginning and end of Course B (winter term of their second year of study), and again at the beginning of the fall term in their 3rd year of study. Cohort 2 was assessed at the beginning of Course A (fall term of second year), and at the beginning of Course B (winter term of second year). Detailed reports are discussed in publications related to this project ${ }^{8,16}$. Survey responses were collected from 68 to 97 participants; Cohort 1 includes 104 students, and Cohort 2 includes 109 students. The same survey was administered. In addition to rating their confidence level, students were asked to organize a list of problem-solving steps according to their perception of which (of the steps listed below) is the easiest and which is the most difficult step. One sampled question is reported below. 


\section{Which of the following steps is the the most difficult for you when solving a}

problem?

- 1. Read the problem;

- 2. Connect problem to theory;

- 3. Make a diagram/picture of the problem;

- 4. State assumptions;

- 5. Develop mathematical equations;

- 6. Find values in tables/charts;

- 7. Perform computations;

- 8. Check numerical answer/unit consistency;

- 9. Other.

Results from this survey are combined with classroom observations and course evaluations to assess the effectiveness of the pedagogical approach and to adjust how the curriculum for Courses $\mathrm{A}$ and $\mathrm{B}$ is to be delivered in the following years. To support the discussion developed in the present contribution, selected data from results presented in a manuscript in preparation ${ }^{16}$ are reported in Figure 2. These preliminary results show that students perceive steps 2 and 5 (i.e., connecting the problem to theory and developing mathematical equations) as the most difficult steps in problem-solving. The proportion of students reporting step 5, "Developing mathematical equations" as their most difficult step tends to go down during a given semester, but goes back up when moving to third year; this evolution is highlighted in a zoomed-in portion of Figure 2 shown in Figure 3. 


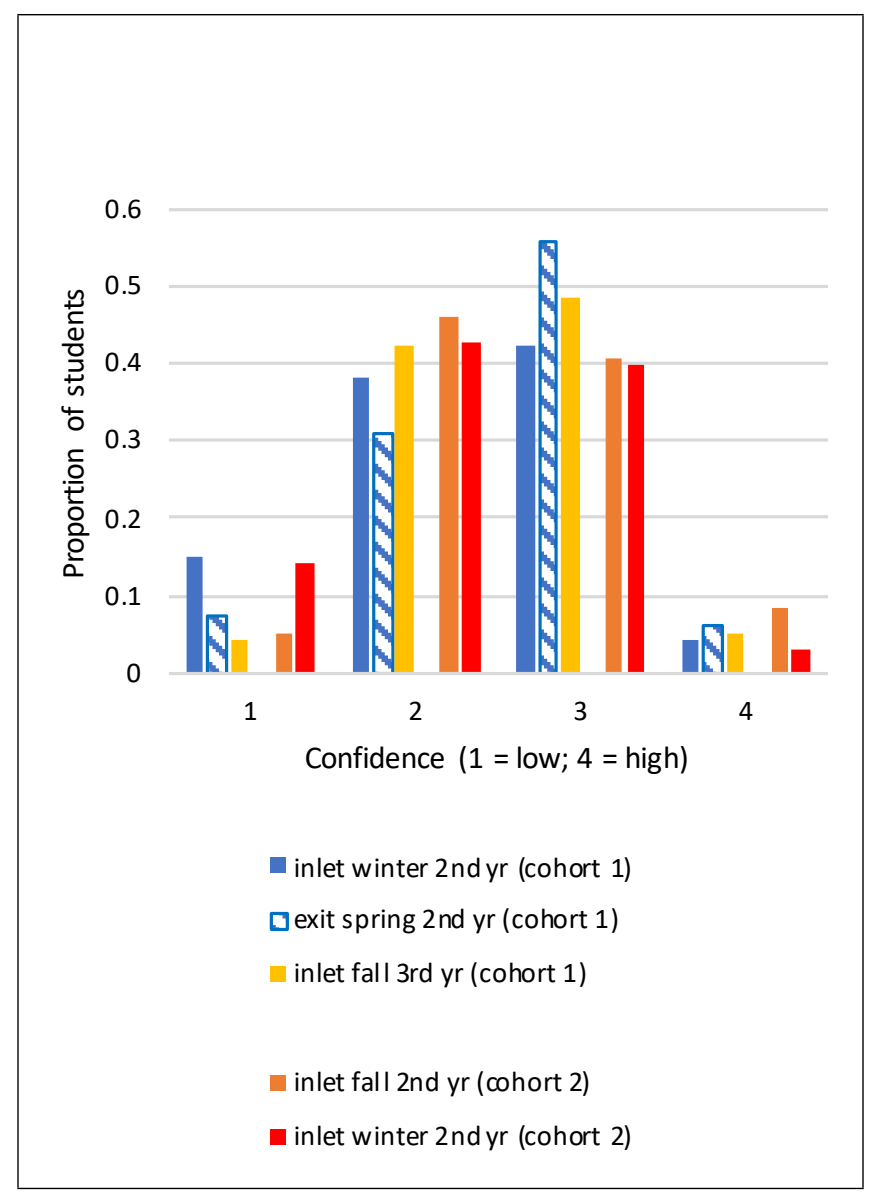

Figure 2: Most difficult task in solving problem ${ }^{16}$

As students are progressing through one course, their level of confidence generally increases; however, their central apprehensions about solving problems are consistent and persistent, i.e., developing the appropriate mathematical framework and relating the correct mathematical framework to theory, are two elements where they feel something is lacking. Moreover, and this may be a direct consequence of their perceived lack of acquired mathematical competency, their level of confidence drops when progressing from one course to another. This calls for extending the current problem-solving project in theoretical courses beyond the second year to promote continuity and long-term positive effects.

As this project is in its second year of research, collected data is insufficient to draw strong conclusions. However, this infomation has been used to improve teaching and develop new teaching activities to improve students' skills in solving chemical engineering problems. To the knowledge of the authors, no study has been reported in the literature on the specific topic of students' perception of their problem-solving skills fduring their chemical engineering studies. As the current project is developing in the next few years, it is hoped that gathered data would point out to more general information and call for more direct action in teaching over the entire chemical engineering curriculum. 


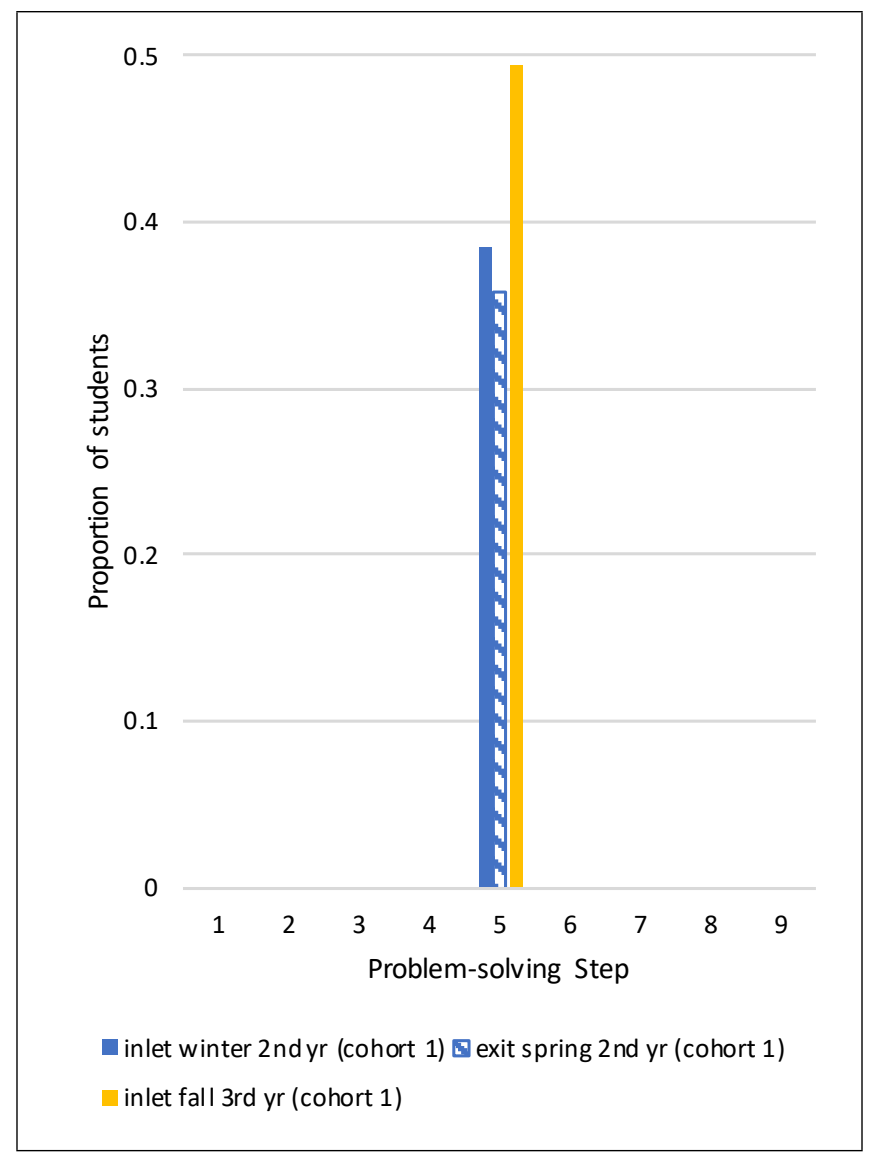

Figure 3: Most difficult task in solving problem — Mathematics — Evolution for cohort 1

\section{Conclusions}

This contribution reports new evidence that a common approach to teach problem-solving is valuable at an early stage of undergraduate engineering studies. This contribution, based on the theory introduced previously ${ }^{8,16}$, advocates that specializing a problem-solving methodology for chemical engineering students can be beneficial in second year, but needs to be extended to upper-year instruction to consolidate student understanding of problem-solving. Ideas supporting the concept map as a curriculum alignment tool are discussed. In the next stage of this project, instructors of Courses A and B will seek to extend the common approach beyond second year, by inviting upper-year instructors to adopt the proposed concept map in designing their instruction activities. Finally, new teaching activities and approaches will be considered in the delivery of material throughout the curriculum to foster students' ownership of an appropriate and effective problem-solving methodology, i.e., by guiding the students in developing long-lasting problem-solving habits beyond second year. Another idea to explore in the present context is to study the impact of specific teaching activities on students' perception of their problem-solving activities, for example the impact of video examples ${ }^{19}$. 


\section{References}

[1] D. H. Jonassen. Toward a design theory of problem solving. Educational Technology Research and Development, 48(4):63-85, 2000.

[2] D. H. Jonassen. Learning to Solve Problems: An Instructional Design Guide. Instructional Technology and Training Series. Pfeiffer, San Francisco, CA, 2004.

[3] D. H. Jonassen. Learning to Solve Problems: A Handbook for Designing Problem-solving Learning Environment. Routhledge, New York, NY, 2011.

[4] D. R. Woods, A. N. Hrymak, R. R. Marshall, P. E. Woods, C. M. Crowe, T. W. Hoffman, J. D. Wright, P. A. Taylor, K. A. Woodhouse, and C. G. K. Bouchard. Developing problem solving skills: The McMaster problem solving program. Journal of Engineering Education, 86(2):75-91, 1997.

[5] P. C. Wankat and F. S. Oreovicz. Teaching Engineering. Purdue University Press, 2nd edition, 2015.

[6] D. R. Woods. An evidence-based strategy for problem solving. Journal of Engineering Education, 89(4): 443-459, 2000.

[7] G. Polya. How to Solve it: A New Aspect of Mathematical Method. Princeton University Press, Princeton, NJ, 2nd edition, 1957.

[8] L. Meunier and N. Hudon. Translating problem statements into actionable solution procedure: Developing problem-solving skills in chemical engineering second-year students. In Proceedings of the 2019 Conference of the Canadian Engineering Education Association, Ottawa, Canada, 2019.

[9] F. H. Reardon. Developing problem-solving skills in thermodynamics courses. In Proceeedings of the 2001 American Society for Engineering Education Annual Conference, 2001.

[10] W. M. McCracken and W. C. Newstetter. Text to diagram to symbol: Representational transformatioms in problem-solving. In Proceedings of the 31st ASEE/IEEE Frontiers in Education Conference, pages F2G-13-17, Reno, NV, 2001.

[11] T. A. Litzinger, P. van Meter, C. M. Firetto, L. J. Passmore, C. B. Masters, S. R. Turns, G. L. Gray, F. Costanzo, and S. E. Zappe. A cognitive study of problem solving in statics. Journal of Engineering Education, 99(4): 337-353, 2010.

[12] P. S. Steif, J. M. Lobue, L. B. Kara, and A. L. Fay. Improving problem solving performance by inducing talk about salient problem features. Journal of Engineering Education, 99(2):135-142, 2010.

[13] P. Rivera-Reyes and L. C. Pérez. Abstraction and problem solving in an uncergraduate electrical engineering circuits course. In Proceedings of the 2016 IEEE Frontiers in Education Conference, Erie, PA, 2016.

[14] H. Sevian, S. Bernholt, G. A. Szteinberg, S. Auguste, and L. C. Pérez. Use of representation mapping to capture abstraction in problem solving in different courses in chemistry. Chemistry Education Research and Practice, 16:429-446, 2015.

[15] J. Harlim and I. Belski. Long-term innovative problem solving skills: Redefining problem solving. International Journal of Engineering Education, 29(2):280-290, 2013.

[16] L. Meunier and N. Hudon. Implementing a common approach to problem-solving in the second year of chemical engineering. In Proceedings of the 2020 Conference of the Canadian Engineering Education Association, Montréal, Canada, 2020.

[17] J. Cronjé. Paradigm regained: Toward integrating objectivism and constructivism in instructional design and the learning sciences. Educational Technology Research and Development, 54(4):387-416, 2006.

[18] S. I. Sandler. Chemical, Biochemical, and Engineering Thermodynamics. John Wiley and Sons, Hoboken, NJ, 5th edition, 2017. 
[19] R. Moeno, M. Reisslein, and G. M. Delgoda. Toward a fundamental understanding of workd example instruction: Impact of means-ends practice, backward/forward fading, and adaptivity. In Proceedings of the 36th ASEE/IEEE Frontiers in Education Conference, pages S3D-5-10, San Diego, CA, 200.

\section{A Thermodynamic Illustrative Problem and Solution}

The following example is taken from a second-year thermodynamic course and is inspired by a similar question in a classical reference ${ }^{18}$. The question is to decide if a proposed process is thermodynamically feasible, by testing the first and second laws of thermodynamics. In this example, students are first asked to draw a diagram for data book-keeping and then are then asked to develop mass, energy, and entropy balances. An extension is explicitly asked to the students in part (d), modifying an earlier assumption. The problem and the solution provided to students are presented without any modification.

An inventor is proposing a black box device using electrical power to compress and separate air adiabatically. The device operates on an input stream of air at $P_{\text {in }}=100 \mathrm{kPa}$ and $T_{\mathrm{in}}=298 \mathrm{~K}$. This input stream is split into two equal molar streams with pressures $P_{\text {out }, 1}=P_{\text {out }, 2}=125 \mathrm{kPa}$ at $T_{\text {out }, 1}=368 \mathrm{~K}$ and $T_{\text {out }, 2}=288 \mathrm{~K}$, respectively. The claim is made that, at steady-state, work is added, on a molar basis, at a rate of $\dot{W}_{B}=900$ $\mathrm{kJ} / \mathrm{kmol}$ of air flowing through the device. Assuming that air behaves like an ideal gas with constant heat capacity $C_{P}^{*}=30 \mathrm{~kJ} /(\mathrm{kmol} . \mathrm{K})$, determine if the process is feasible. The enthalpy can be approximated by a linear function of temperature, i.e., $h(T)=C_{P}^{*} \cdot T$ and entropy can be approximated as a function of temperature and pressure, i.e., $s(T, P)=C_{P}^{*} \ln (T)-R \ln (P)$, where $R=8.314 \mathrm{~kJ} /(\mathrm{kmol} . \mathrm{K})$.

- a. Sketch and label the process.

- b. Develop mass, energy, and entropy balances for this evolution process. State all relevant assumptions

- c. Solve the balances numerically and determine if the process thermodynamically feasible.

- $\mathrm{d}$. When testing the system, you realize that the process cannot be operated adiabatically, i.e., heat is lost at the boundary of the process $\left(\dot{Q}_{B}>0\right)$. How would you change the process inlet to make the process feasible and keep the outlet streams as specified?

(a) A sketch of the proposed process is given in Figure 4.

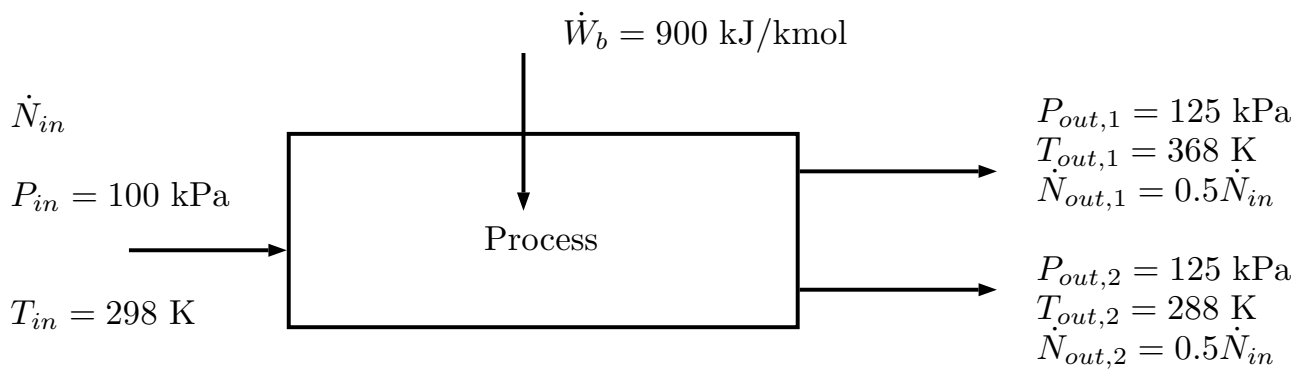

Figure 4: Diagram — Example Problem 
(b) Mass balance (in moles):

At steady-state, no accumulations, the mass balance is of the form:

$$
\frac{d y^{1}}{d t}=\dot{N}_{\text {in }}-\dot{N}_{\text {out }, 1}-\dot{N}_{\text {out }, 2}
$$

and since

$$
\dot{N}_{\text {out }, 1}=\dot{N}_{\text {out }, 2}=\frac{1}{2} \dot{N}_{\text {in }}
$$

one can verify that mass is conserved for the process.

Energy balance: (in $\mathrm{kJ} / \mathrm{kmol}$ of air)

At steady-state, and assuming the process is adiabatic $\left(\dot{Q}_{B}=0\right)$, one has

$$
\frac{d z^{\prime}}{d t}=\dot{E}_{\text {in }}-\dot{E}_{\text {out }} \pm \dot{\mathscr{Q}}_{b}^{0} \pm \dot{W}_{b}
$$

Further, if kinetic and potential energy variations can be neglected, energy balance becomes

$$
0=\dot{N}_{\text {in }} h_{\text {in }}-\frac{\dot{N}_{\text {in }}}{2} h_{\text {out }, 1}-\frac{\dot{N}_{\text {in }}}{2} h_{\text {out }, 2}+900 \dot{N}_{\text {in }} .
$$

Since we have an ideal gas, and with the linear property on $h$ given in the question, we can rewrite (in Joules)

$$
0=C_{P}^{*} T_{\text {in }}-\frac{1}{2} C_{P}^{*} T_{\text {out }, 1}-\frac{1}{2} C_{P}^{*} T_{\text {out }, 2}+900
$$

in $\mathrm{kJ} / \mathrm{kmol}$ air.

Entropy balance:

At steady-state, and assuming the process is adiabatic $\left(\dot{Q}_{B}=0\right)$, the entropy balance is:

$$
\begin{aligned}
\frac{d S^{0}}{d t} & =\dot{S}_{\text {in }}-\dot{S}_{\text {out }} \pm \frac{\dot{Q} / b}{T_{b}}+\dot{\sigma}_{\mathrm{S}} \\
0 & =\dot{N}_{\text {in }}\left(s_{\text {in }}-\frac{1}{2} s_{\text {out }, 1}-\frac{1}{2} s_{\text {out }, 2}\right)+\dot{S}_{\text {gen }} \\
\dot{\sigma}_{\mathrm{S}} & =\frac{\dot{N}_{\text {in }}}{2}\left(s_{\text {out }, 1}-s_{\text {in }}+s_{\text {out }, 2}-s_{\text {in }}\right) .
\end{aligned}
$$

Since we have assumed that we have an ideal gas, we can rewrite as

$$
\begin{aligned}
\dot{\sigma}_{\mathrm{S}} & =\frac{\dot{N}_{\text {in }}}{2}\left(C_{P}^{*} \ln \left(\frac{T_{\text {out }, 1}}{T_{\text {in }}}\right)-R \ln \left(\frac{P_{\text {out }, 1}}{P_{\text {in }}}\right)+C_{P}^{*} \ln \left(\frac{T_{\text {out }, 2}}{T_{\text {in }}}\right)-R \ln \left(\frac{P_{\text {out }, 2}}{P_{\text {in }}}\right)\right) \\
& =\frac{\dot{N}_{\text {in }}}{2}\left(C_{P}^{*} \ln \left(\frac{T_{\text {out }, 1} T_{\text {out }, 2}}{T_{\text {in }}^{2}}\right)-R \ln \left(\frac{P_{\text {out }, 1} P_{\text {out }, 2}}{P_{\text {in }}^{2}}\right)\right) .
\end{aligned}
$$

(c) Numerical computations: 
We already checked that mass is conserved in the process. We need to check numerically that the first law holds, $i . e$., that energy is conserved. From

$$
0=C_{P}^{*} T_{\text {in }}-\frac{1}{2} C_{P}^{*} T_{\text {out }, 1}-\frac{1}{2} C_{P}^{*} T_{\text {out }, 2}+900,
$$

with units of $\mathrm{kJ} / \mathrm{kmol}$ air, and using the numerical values, one can compute

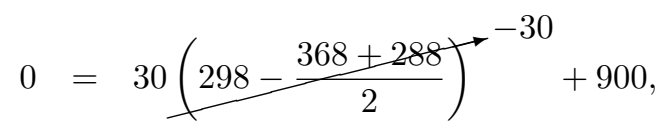

and energy conservation is verified.

From the equations

$$
\dot{\sigma}_{S}=\frac{\dot{N}_{\mathrm{in}}}{2}\left(C_{P}^{*} \ln \left(\frac{T_{\mathrm{out}, 1} T_{\mathrm{out}, 2}}{T_{\mathrm{in}}^{2}}\right)-R \ln \left(\frac{P_{\mathrm{out}, 1} P_{\mathrm{out}, 2}}{P_{\mathrm{in}}^{2}}\right)\right)
$$

and we can compute

$$
\begin{aligned}
\frac{\dot{\sigma}_{S}}{\dot{N}_{\mathrm{in}}} & =\frac{1}{2}\left(30 \ln \left(\frac{368 \cdot 288}{298 \cdot 298}\right)-8.314 \ln \left(\frac{125 \cdot 125}{100 \cdot 100}\right)\right) \\
& =0.8 \frac{\mathrm{kJ}}{\mathrm{K} \cdot \mathrm{kmol}} \geq 0
\end{aligned}
$$

and hence, the second law is verified.

The process is thus possible.

(d) If $\dot{Q}_{b}>0$, the second law is still verified (i.e., we would add a $\frac{\dot{Q}_{b}}{T_{b}}>0$ term to the right hand side of the $\dot{\sigma}_{S}$ expression). However, more energy is needed to keep the energy balance consistent. Hence, one needs to increase the amount of energy in the inlet stream (for example, by increasing the inlet temperature). Also acceptable would be to increase the power applied to the compressor $\dot{W}_{b}$ to match the heat loss. 